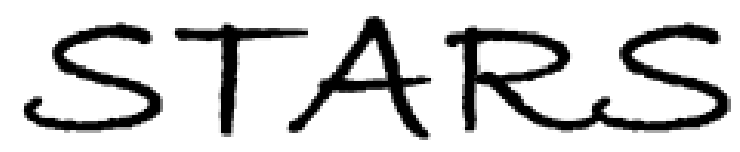

University of Central Florida

STARS

$1-1-1998$

\title{
Linear and nonlinear optical properties of $\mathrm{KNbO3}$ ridge waveguides
}

Tomas Pliska

University of Central Florida

Daniel Fluck

Peter Günter

Lutz Beckers

Christoph Buchal

Find similar works at: https://stars.library.ucf.edu/facultybib1990

University of Central Florida Libraries http://library.ucf.edu

This Article is brought to you for free and open access by the Faculty Bibliography at STARS. It has been accepted for inclusion in Faculty Bibliography 1990s by an authorized administrator of STARS. For more information, please contact STARS@ucf.edu.

\section{Recommended Citation}

Pliska, Tomas; Fluck, Daniel; Günter, Peter; Beckers, Lutz; and Buchal, Christoph, "Linear and nonlinear optical properties of KNbO3 ridge waveguides" (1998). Faculty Bibliography 1990s. 2404.

https://stars.library.ucf.edu/facultybib1990/2404

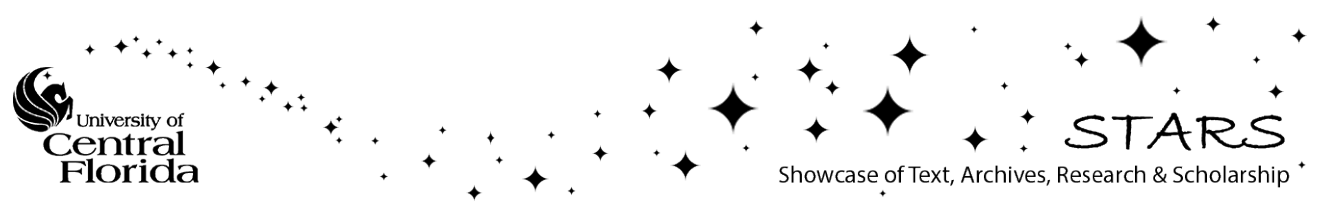




\section{Linear and nonlinear optical properties of $\mathrm{KNbO}_{3}$ ridge waveguides}

Cite as: Journal of Applied Physics 84, 1186 (1998); https://doi.org/10.1063/1.368184

Submitted: 04 February 1998 . Accepted: 23 April 1998 . Published Online: 17 July 1998

Tomas Pliska, Daniel Fluck, Peter Günter, Lutz Beckers, and Christoph Buchal

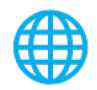

\section{ARTICLES YOU MAY BE INTERESTED IN}

The influence of femtosecond laser wavelength on waveguide fabrication inside fused silica Applied Physics Letters 110, 161109 (2017); https://doi.org/10.1063/1.4981124

Continuously controllable optical band gap in orthorhombic ferroelectric $\mathrm{KNbO}_{3}-\mathrm{BiFeO}_{3}$ ceramics

Applied Physics Letters 110, 172902 (2017); https://doi.org/10.1063/1.4982600

Multifunctional surfaces produced by femtosecond laser pulses

Journal of Applied Physics 117, 033103 (2015); https://doi.org/10.1063/1.4905616

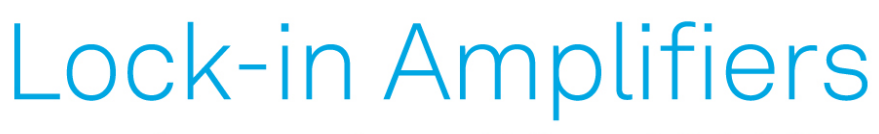

... and more, from DC to $600 \mathrm{MHz}$

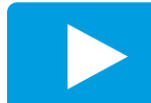

Watch 


\title{
Linear and nonlinear optical properties of $\mathrm{KNbO}_{3}$ ridge waveguides
}

\author{
Tomas Pliska, ${ }^{\text {a) }}$ Daniel Fluck, and Peter Günter \\ Nonlinear Optics Laboratory, Institute of Quantum Electronics, Swiss Federal Institute of Technology, \\ ETH Hönggerberg, CH-8093 Zürich, Switzerland \\ Lutz Beckers and Christoph Buchal \\ Institut für Schicht-und Ionentechnik, Forschungszentrum (KFA), D-52425 Jülich, Germany
}

(Received 4 February 1998; accepted for publication 23 April 1998)

\begin{abstract}
Ridged channel waveguides in $\mathrm{KNbO}_{3}$ were produced using $\mathrm{He}^{+}$ion implantation, photolithographic masking, and subsequent $\mathrm{Ar}^{+}$ion sputtering. We investigated the linear and nonlinear optical characteristics of the waveguides. The effective mode indices are derived from the refractive index profiles using the effective index method. The losses are investigated as a function of wavelength and of the geometrical parameters channel width and ridge height. A minimum loss of $2 \mathrm{~dB} \mathrm{~cm}^{-1}$ is measured at a wavelength of $0.633 \mu \mathrm{m}$. We investigated the power handling capabilities at visible and near-infrared wavelengths. Second-harmonic generation in these waveguides is studied both theoretically and experimentally with regard to its dependence on the guide fabrication parameters. Phase-matching configurations for blue light second-harmonic generation are evaluated on the basis of the dispersion of the effective mode indices. Overlap integrals are calculated on the basis of the field distributions derived from the refractive index profiles. The minimum effective guide cross section is $25 \mu \mathrm{m}^{2}$. A continuous-wave second-harmonic output power of $14 \mathrm{~mW}$ at $438 \mathrm{~nm}$ was obtained with an in-coupled fundamental power of $340 \mathrm{~mW}$ in a $0.73 \mathrm{~cm}$ long waveguide, yielding a normalized internal conversion efficiency of $25 \% \mathrm{~W}^{-1} \mathrm{~cm}^{-2}\left(13 \% \mathrm{~W}^{-1}\right)$. This corresponds to an improvement by a factor of 3 compared to the best results reported for $\mathrm{KNbO}_{3}$ waveguides up to now. (C) 1998 American Institute of Physics. [S0021-8979(98)00715-4]
\end{abstract}

\section{INTRODUCTION}

Potassium niobate $\left(\mathrm{KNbO}_{3}\right)$ has been shown to be highly suitable for electro-optical and nonlinear optical applications due to its large electro-optical coefficients ${ }^{1,2}$ and high nonlinear optical susceptibilities. ${ }^{3,4}$ In particular, the favorable second-harmonic phase-matching properties down to $850 \mathrm{~nm}$ without the need of periodic poling ${ }^{5}$ make $\mathrm{KNbO}_{3}$ an attractive material for efficient second-harmonic generation (SHG) of near-infrared laser radiation. $\mathrm{KNbO}_{3}$ is one of the candidate materials for the realization of a compact blue laser source for applications such as optical data storage, printing, and spectroscopy.

Second-harmonic generation with diode lasers in $\mathrm{KNbO}_{3}$ was first reported in Ref. 6. The development of high power master-oscillator power-amplifier (MOPA) diode lasers has allowed the demonstration of efficient second-harmonic generation in a single-pass geometry using bulk $\mathrm{KNbO}_{3}$ crystals. $^{7,8}$ Very efficient second-harmonic generation has been achieved in resonant devices. ${ }^{9,10}$ However, resonant schemes suffer from the disadvantage that they need active stabilization of the diode laser and doubling cavity that must be provided by an electronic feedback loop.

\footnotetext{
${ }^{a)}$ Present address: Center for Research and Education in Optics and Lasers (CREOL), University of Central Florida, P.O. Box 162700, 4000 Central Florida Blvd., Orlando, FL 32816-2700; electronic mail: pliska @ mail.creol.ucf.edu
}

Waveguide devices are an alternative approach as they offer the possibility of achieving high conversion efficiencies in a simple single-pass scheme. ${ }^{11}$ Second-harmonic generation has been demonstrated in various ferroelectric waveguides, including lithium niobate $\left(\mathrm{LiNbO}_{3}\right),{ }^{12,13}$ lithium tantalate $\left(\mathrm{LiTaO}_{3}\right),{ }^{14}$ and potassium titanyl phosphate $\left[\mathrm{KTiOPO}_{4},(\mathrm{KTP})\right] .{ }^{15,16}$ Recently, results were reported on Cerenkov-type and guided-mode frequency doubling in $\mathrm{KNbO}_{3}$ waveguides. ${ }^{17,18}$

Permanent waveguides in $\mathrm{KNbO}_{3}$ formed by ion implantation were produced for the first time by Bremer et al. ${ }^{19}$ Subsequent reports concentrated on index depth profiles. ${ }^{20,21}$ The influence of the ion dose on loss in planar waveguides was investigated by Strohkendl et al. ${ }^{22}$ The first embeddedtype channel waveguides in $\mathrm{KNbO}_{3}$ formed by ion irradiation using a multiple energy implantation procedure were reported by Fluck et al. ${ }^{23}$ The continuous progress of guide fabrication technology has allowed the fabrication of channel waveguides by an alternative single energy irradiation process. ${ }^{18}$

In this article we will discuss a new technology that permits the manufacture of ridge waveguides in $\mathrm{KNbO}_{3}$ with excellent nonlinear optical properties. Results on the effective mode index evaluation, attenuation characteristics, power handling capabilities, and second-harmonic generation will be presented. 


\section{THEORY OF SECOND-HARMONIC GENERATION IN CHANNEL WAVEGUIDES}

Here we will briefly summarize the relevant relations for second-harmonic generation in channel waveguides. We start with the definition of the nonlinear optical susceptibility $d_{i j k}$ that relates the nonlinear polarization component $P_{i}(2 \omega)$ to the driving fields $E_{j}(\omega)$ and $E_{k}(\omega)$,

$$
P_{i}(2 \omega)=\epsilon_{0} d_{i j k} E_{j}(\omega) E_{k}(\omega) .
$$

The derivation of the coupled-mode equation for secondharmonic generation is shown, for example, in Ref. 24. For type I phase matching $(j=k)$ under the nondepleted pump approximation (no depletion of the fundamental beam due to frequency conversion) we find for the second-harmonic power at the guide end,

$$
P_{2 \omega}(L)=\frac{2 \omega^{2}}{\epsilon_{0} c^{3}} \frac{d_{\text {mat }}^{2}}{N_{2 \omega} N_{\omega}^{2}} L^{2} P_{\omega}^{2}(0) h\left(\Delta \beta, \alpha_{\omega}, \alpha_{2 \omega}, L\right) \Gamma,
$$

where $\omega$ denotes the angular frequency of light at the fundamental wavelength, $\epsilon_{0}$ the permittivity constant, $c$ the speed of light, $d_{\text {mat }}$ the effective nonlinear optical coefficient of the material, $N$ the effective mode index, $L$ the guide length, and $P_{\omega}(0)$ the fundamental power coupled into guide. The fundamental and second-harmonic waves can be carried in modes of different order. The phase-mismatch function $h$ is given by

$$
h\left(\Delta \beta, \alpha_{\omega}, \alpha_{2 \omega}, L\right)=\exp \left[-\left(\frac{\alpha_{2 \omega}}{2}+\alpha_{\omega}\right) L\right] \frac{[\sinh (\Delta \alpha L / 2) \cos (\alpha \beta L / 2)]^{2}+[\cosh (\Delta \alpha L / 2) \sin (\Delta \beta L / 2)]^{2}}{\left[\left(\Delta \alpha^{2}+\Delta \beta^{2}\right) L^{2} / 4\right]}
$$

$$
\begin{aligned}
& \Delta \beta=\beta_{2 \omega}-2 \beta_{\omega}, \\
& \beta_{i}=\frac{2 \pi}{\lambda_{i}} N_{i}, \\
& \Delta \alpha=\frac{\alpha_{2 \omega}}{2}-\alpha_{\omega},
\end{aligned}
$$

where $\beta_{i}$ denotes the mode propagation constant, and $\alpha_{i}$ the power attenuation constant. In the case of phase matching, $\Delta \beta=0, h$ reduces to

$$
\begin{aligned}
& h\left(\alpha_{\omega}, \alpha_{2 \omega}, L\right) \\
& \quad=\exp \left[-\left(\frac{\alpha_{2 \omega}}{2}+\alpha_{\omega}\right) L\right] \frac{[\sinh (\Delta \alpha L / 2)]^{2}}{(\Delta \alpha L / 2)^{2}} .
\end{aligned}
$$

Equation (4) describes the reduction of the secondharmonic power due to the linear losses. The overlap integral $\Gamma$ is given by

$$
\Gamma=\left(\iint \bar{d}(x, y) \phi_{\omega}^{2}(x, y) \phi_{2 \omega}(x, y) d x d y\right)^{2}
$$

under the normalization condition

$$
\iint \phi_{i}^{2}(x, y) d x d y=1 .
$$

$\phi_{i}(x, y)$ denotes the distribution of the main field component of the fundamental and second-harmonic wave, respectively, i.e., $\phi=E_{x}$ for the transverse electric (TE) mode and $\phi$ $=H_{x}$ for the transverse magnetic (TM) mode. The normalized nonlinear optical coefficient $\bar{d}$ of the waveguide is defined by

$$
\bar{d}(x, y)=\frac{d_{w g}(x, y)}{d_{\text {mat }}},
$$

where $d_{w g}(x, y)$ denotes the nonlinearity of the waveguide. $d_{w g}$ may spatially vary while we assume that $d_{\text {mat }}$ is a constant material parameter.
We define a normalized conversion efficiency (secondharmonic figure-of-merit) on the basis of Eq. (2) by

$$
\eta \equiv \frac{1}{L^{2}} \frac{P_{2 \omega}(L)}{P_{\omega}^{2}(0)}=\frac{2 \omega^{2}}{\epsilon_{0} c^{3}} \frac{d_{\mathrm{mat}}^{2}}{N_{2 \omega} N_{\omega}^{2}} h \Gamma .
$$

Notice that $\eta$ is normalized to the in-coupled fundamental power. For an ideal lossless waveguide we find $h=1$ according to Eq. (4) so that the corresponding normalized conversion efficiency is

$$
\eta_{0}=\frac{2 \omega^{2}}{\epsilon_{0} c^{3}} \frac{d_{\mathrm{mat}}^{2}}{N_{2 \omega} N_{\omega}^{2}} \Gamma
$$

Normalization to the fundamental power $P_{\omega}(L)$ at the end of the guide, defined by

$$
\tilde{\eta} \equiv \frac{1}{L^{2}} \frac{P_{2 \omega}(L)}{P_{\omega}^{2}(L)}=\frac{2 \omega^{2}}{\epsilon_{0} c^{3}} \frac{d_{\mathrm{mat}}^{2}}{N_{2 \omega} N_{\omega}^{2}} h \Gamma \exp \left(2 \alpha_{\omega} L\right),
$$

may yield useful information if $\alpha_{2 \omega}=2 \alpha_{\omega}$. In this case, we find $h=\exp \left(-2 \alpha_{\omega} L\right)$ and by comparing Eqs. (9) and (10)

$$
\eta_{0}=\tilde{\eta}
$$

In other words, for a waveguide with $\alpha_{2 \omega} \approx 2 \alpha_{\omega}$, the measured conversion efficiency normalized to the fundamental power at the guide end is equal to the normalized conversion efficiency of the same waveguide without propagation loss. ${ }^{13}$ Thus, $\tilde{\eta}$ provides an experimental measure of the maximum attainable conversion efficiency. However, to reflect the nonlinear properties of a waveguide, we prefer to indicate $\eta$, because $\tilde{\eta}$ tends to overestimate the conversion efficiency in the presence of guide attenuation. 
(a)
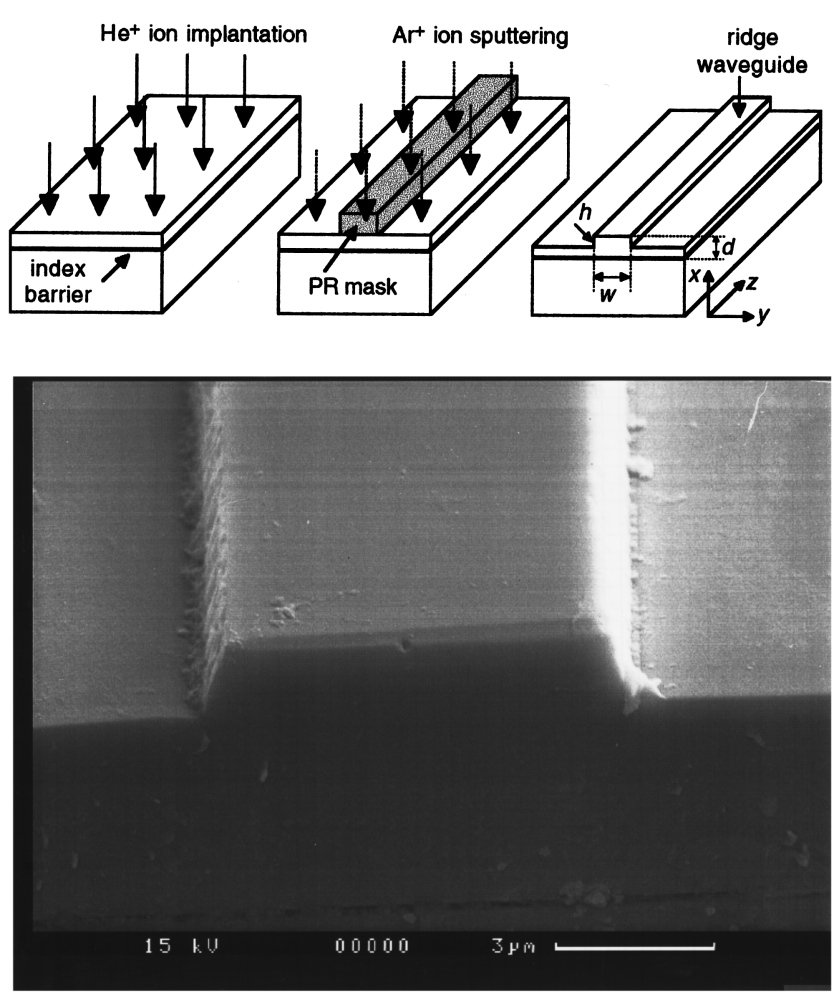

(d)

FIG. 1. Schematic of the ridge waveguide fabrication process. (a) Formation of a planar waveguide in a $\mathrm{KNbO}_{3}$ single crystal by $\mathrm{He}^{+}$ion implantation. (b) Formation of a photoresist mask and subsequent sputtering with $\mathrm{Ar}^{+}$ ions from a plasma ion source. (c) Resulting ridge waveguide with thickness $d$, width $w$, and ridge height $h$. (d) Scanning electron micrograph of a $\mathrm{KNbO}_{3}$ ridge waveguide with $h=1.3 \mu \mathrm{m}, w=7.0 \mu \mathrm{m}$, and $d=5.3 \mu \mathrm{m}$.

\section{WAVEGUIDE FABRICATION}

The ridge waveguide fabrication procedure is schematically displayed in Fig. 1. The first step [Fig. 1(a)], irradiation of a $\mathrm{KNbO}_{3}$ single crystal with $\mathrm{MeV} \mathrm{He}{ }^{+}$ions, leads to the formation of a refractive index barrier buried below the crystal surface. This means that a planar waveguide is obtained. In the next step [Fig. 1(b)], a photoresist (PR) mask defining strips of a few micrometers width is formed on top of the planar waveguide by photolithography. Subsequently, $\mathrm{Ar}^{+}$ ion sputtering in a plasma ion source is used to etch the planar waveguide in the unshielded regions. The resulting ridge waveguide with guide thickness $d$, guide width $w$, and ridge height $h$ is shown in Fig. 1(c). A scanning electron micrograph of the front face of a ridge waveguide with $h$ $=1.3 \mu \mathrm{m}, w=7.0 \mu \mathrm{m}$, and $d=5.3 \mu \mathrm{m}$ is shown in Fig. $1(\mathrm{~d})$.

Both ion implantation and ion sputtering allow accurate adjustment of the processing parameters. Therefore, the fabrication process is very reproducible. The energy of the $\mathrm{He}^{+}$ ions, and thus the guide thickness, can be controlled to an accuracy of better than $1 \%$. Similarly, the photolithographic masking and dry etching allow one to precisely pattern ridges to a desired size.

The waveguides used in this work were all fabricated with $\mathrm{He}^{+}$ions of $E=2.3 \mathrm{MeV}$ energy resulting in a planar waveguide of $d=5.3 \mu \mathrm{m}$ thickness. ${ }^{25}$ Except for the four waveguides used for the measurement shown in Fig. 6, which were implanted with an ion dose of $D=2.5$ $\times 10^{15} \mathrm{~cm}^{-2}$, all waveguides were fabricated with $D=1.5$ $\times 10^{15} \mathrm{~cm}^{-2}$. The etch rate of the $\mathrm{Ar}^{+}$ion sputtering process was $1.7 \mathrm{~nm} / \mathrm{min}$. For optical characterization waveguides with a ridge height of up to $h=0.9 \mu \mathrm{m}$ and a maximum width of $w=7.6 \mu \mathrm{m}$ were used.

After implantation and ridge etching the waveguides were annealed at $180^{\circ} \mathrm{C}$ for about $10 \mathrm{~h}$ to decrease the optical loss due to defect centers generated during the $\mathrm{He}^{+}$ion irradiation. ${ }^{26}$ Finally, the end faces of the crystal were polished to optical quality to allow end-fire coupling.

\section{INDEX PROFILES AND EFFECTIVE MODE INDICES}

Knowledge of the refractive index profiles and the corresponding effective mode indices and mode intensity distributions forms the basis for the evaluation of the linear and nonlinear optical characteristics of ion-implanted $\mathrm{KNbO}_{3}$ waveguides, such as attenuation, phase-matching conditions, and overlap integrals. The refractive index modification in $\mathrm{KNbO}_{3}$ due to ion irradiation is thoroughly discussed in Refs. 25, 27, and 28. It was shown that the effective mode indices of planar $\mathrm{KNbO}_{3}$ waveguides can be predicted with an absolute accuracy of better than $\pm 10^{-3}$ as a function of ion energy and dose.

To calculate the effective mode indices of the ridged channel waveguides, we use the effective index method. ${ }^{29,30}$ The method can be outlined as follows. First, the ridge and its lateral surrounding regions are viewed as three separate planar waveguides. (Notice that in this channel guide type the lateral surrounding region of the ridge supports guided modes too.) The effective mode indices of these planar waveguides are calculated for each region based on the corresponding irradiation parameters. Next, the structure is considered from a bird's-eye view as a symmetric planar waveguide with a top-hat index profile where the refractive indices are replaced by the corresponding effective indices of the ridge and surround regions. The evaluation of the effective index $N$ of this equivalent guide concludes the procedure.

Experimentally, the effective indices are determined by a method which we refer to as the radiation angle method. Prism coupling which is often used to measure the mode spectra of planar waveguides does not allow efficient coupling into channel waveguides. Therefore, to measure the effective mode indices of our channel waveguides, we make use of the inherent property of barrier waveguides formed by ion implantation that part of the light leaks from the waveguide through the barrier into the substrate. The light carried in mode $(m n)$ tunnelling through the barrier is coherently radiated into the substrate under an internal angle $\theta_{m n}$ which is related to the effective mode index $N_{m n}$ by

$$
N_{m n}=n_{\text {bulk }} \cos \theta_{m n},
$$

where $n_{\text {bulk }}$ denotes the refractive index seen by the light in the $\mathrm{KNbO}_{3}$ substrate. Depending on crystal orientation and polarization $n_{\text {bulk }}$ can be a function of $\theta_{m n}$. The angle $\theta_{m n}$ is characteristic for each waveguide mode and becomes larger with increasing mode order. This radiated light from the waveguide appears as a pattern of semicircles if observed on 
(a)

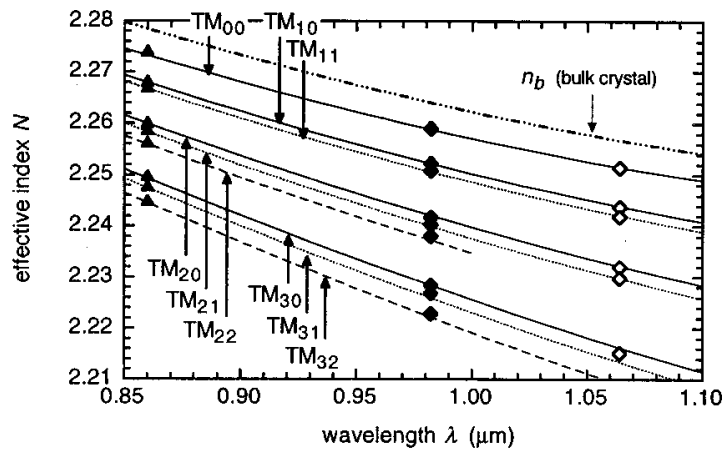

(b)

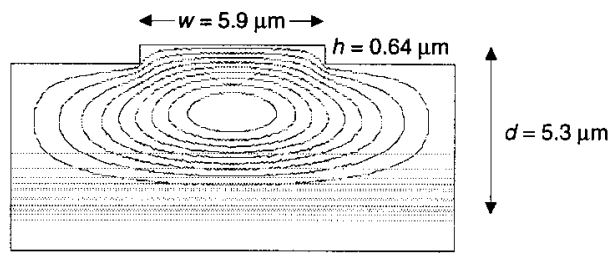

FIG. 2. (a) Wavelength dispersion of the effective mode indices of a ridge waveguide (TM modes with polarization parallel to the crystallographic $b$ axis). The full, dotted, and dashed lines (-, $\cdots,-----)$ indicate the calculated dispersion, and the symbols represent the values measured by the radiation angle method at $0.860 \mu \mathrm{m}(\boldsymbol{\Delta}), 0.982 \mu \mathrm{m}(\diamond)$, and $1.064 \mu \mathrm{m}(\diamond)$. The dash-dotted line $(-\cdots-\cdots-)$ represents the bulk crystal refractive index $n_{b}$. Waveguide parameters: $d=5.3 \mu \mathrm{m}, w=5.9 \mu \mathrm{m}, h=0.64 \mu \mathrm{m}$. (b) Calculated contour plot of the modal field distribution of the waveguide of (a). The horizontal lines indicate the discretization of the index profile.

a screen behind the waveguide. The effective index can be obtained by measuring the radiation angle of each waveguide mode. The absolute accuracy of the method is $10^{-3}$, a value comparable to the accuracy achievable with prism coupling.

We measured the effective mode indices of several channel waveguides using the radiation angle method. Figure 2(a) shows the comparison between the calculated (indicated by lines) and measured wavelength dispersion of the TM-mode indices (polarized along the $b$ axis) of a ridge waveguide with $d=5.3 \mu \mathrm{m}, w=5.9 \mu \mathrm{m}$, and $h=0.64 \mu \mathrm{m}$. All measured effective indices correspond within an accuracy of 8 $\times 10^{-4}$ to the calculated values which provides very good proof that the effective index method is well suited to calculating the effective indices of these ridge waveguides. Notice that the cut-off wavelength for the $\mathrm{TM}_{22}$ mode is about $1 \mu \mathrm{m}$ so it was observed at 0.860 and $0.982 \mu \mathrm{m}$, but not at 1.064 $\mu \mathrm{m}$. Figure 2(b) shows a contour plot of the modal field distribution of the corresponding ridge waveguide. The field distribution was calculated numerically using a variational technique.

\section{WAVEGUIDE ATTENUATION}

Losses in ion-implanted waveguides originate from three sources: scattering, absorption, and tunnelling. ${ }^{31} \mathrm{We}$ have recently reported in detail on losses in ion-implanted $\mathrm{KNbO}_{3}$ waveguides. ${ }^{32} \mathrm{~A}$ semi-empirical model based on simple power-law functions was proposed to describe the attenuation with respect to wavelength, ion dose, guide thickness

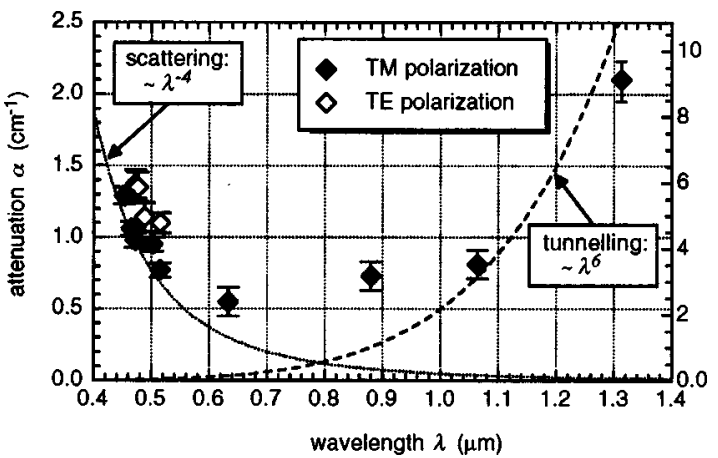

FIG. 3. Attenuation as a function of wavelength of a ridge waveguide. The line represents the approximate trends of scattering and tunneling loss, respectively. Waveguide parameters: $d=5.3 \mu \mathrm{m}, w=2.6 \mu \mathrm{m}, h=0.91 \mu \mathrm{m}$. $(\diamond)$ TM polarization (parallel to $b$ ); $(\diamond)$ TE polarization (parallel to $c$ ).

and guide width. Evaluating the dependence of the loss on these parameters is an essential prerequisite for optimization of the waveguides for parametric processes.

Implantation of $\mathrm{MeV} \mathrm{He}^{+}$ions into a $\mathrm{KNbO}_{3}$ single crystal has an impact on the lattice itself as well as on the refractive index. ${ }^{33}$ Besides the formation of a refractive index barrier, the implantation causes lattice damage in the form of vacancies and interstitials that can act as scattering and absorption centers. The losses due to these defects are dominant at visible wavelengths and increase towards the ultraviolet. As the amount of lattice damage grows with increasing ion dose, absorption and scattering increase towards higher doses. Although annealing at $180{ }^{\circ} \mathrm{C}$ reduces the loss at visible wavelengths considerably ${ }^{26}$ there remains residual loss due to absorption and scattering. These losses could be further reduced by use of a higher annealing temperature. This is, however, prevented by the structural phase transition of $\mathrm{KNbO}_{3}$ at $220{ }^{\circ} \mathrm{C}$.

At near-infrared wavelengths above $0.7 \mu \mathrm{m}$, tunnelling through the refractive index barrier becomes the dominant contribution to the loss, rapidly growing towards longer wavelengths. Reduction of tunnelling at the fundamental wavelength can be achieved by increasing the ion dose which leads to a larger decrease of the refractive index in the barrier and, thus, to a stronger confinement of the waveguide mode. On the other hand, increasing the ion dose causes higher loss at the second-harmonic wavelength. Therefore, optimization of the waveguide for second-harmonic generation is a trade-off between scattering and tunnelling which requires careful evaluation of the appropriate ion dose. Doses between $1.5 \times 10^{15}$ and $2.5 \times 10^{15} \mathrm{~cm}^{-2}$ have been found to be optimum. ${ }^{32}$

In ridge waveguides the coupling of light from the channel guide to the surround modes is an additional source of loss as the surround of the ridge supports guided modes too. The lateral confinement of the mode is expected to increase with growing width and ridge height.

The attenuation as a function of wavelength between 0.4 and $1.4 \mu \mathrm{m}$ of a ridge waveguide $(d=5.3 \mu \mathrm{m}, \quad w$ $=2.6 \mu \mathrm{m}$, and $h=0.91 \mu \mathrm{m})$ is displayed in Fig. 3. The curves indicate the approximate trends of the scattering and tunnelling loss, respectively. The wavelength dependence of 
(a)

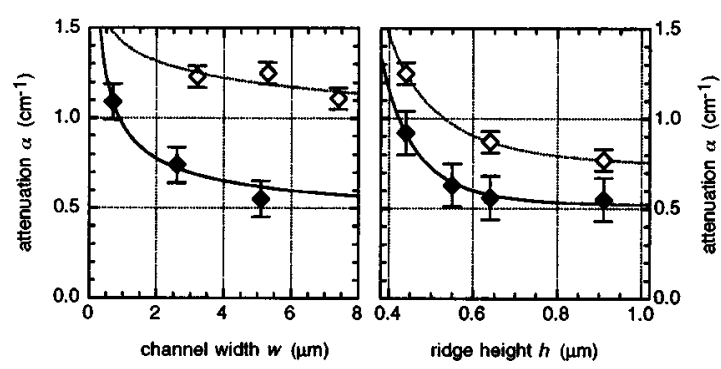

FIG. 4. (a) Attenuation as a function of channel width of ridge waveguides $(d=5.3 \mu \mathrm{m})$ measured at $0.515 \mu \mathrm{m}(\diamond, h=0.44 \mu \mathrm{m})$ and $0.870 \mu \mathrm{m}(\diamond$, $h=0.91 \mu \mathrm{m}$ ). (b) Attenuation as a function of the ridge height measured at $0.515(\diamond)$ and $0.870 \mu \mathrm{m}(\diamond)$. Waveguide parameters: $d=5.3 \mu \mathrm{m} ; w$ $=5.1 \mu \mathrm{m}$. The lines represent fits according to a power-law function.

the loss exhibits the characteristic features discussed above: minimum loss at red wavelengths and an increase towards shorter and longer wavelengths due to scattering and tunnelling, respectively. The loss at blue wavelengths was measured for both TE and TM polarization. The TE modes polarized parallel to $c$ exhibit a slightly higher loss than the TM modes polarized parallel to $b$.

The channel width dependence of the loss, measured at 0.515 and $0.875 \mu \mathrm{m}$, respectively, is shown in Fig. 4(a). Fitted power-law functions are indicated by curves. A series of ridge waveguides was used to investigate the dependence of the loss on the ridge height at wavelengths of 0.515 and $0.870 \mu \mathrm{m}$, shown in Fig. 4(b). Again, the lines indicate curves fitted according to a power-law dependence.

\section{POWER HANDLING CAPABILITIES}

One of the most prominent problems encountered when using ferroelectric oxides, such as $\mathrm{LiNbO}_{3}$ and $\mathrm{LiTaO}_{3}$, as second-harmonic generators is optical damage, in general attributed to light induced refractive index changes. Optical damage can be particularly detrimental at the high optical intensities in waveguides. The effect manifests itself through distortions of the optical field distribution and throughput power fluctuation and decrease, often leading to complete failure of the device after seconds or minutes. Optical damage has been investigated, e.g., in periodically poled annealed proton-exchanged $\mathrm{LiNbO}_{3}$ waveguides, ${ }^{34}$ protonexchanged $\mathrm{LiTaO}_{3}$ waveguides, ${ }^{35}$ and segmented rubidiumexchanged KTP waveguides. ${ }^{34,36}$ Although means to reduce optical damage have been found in the past, the effect is still thought to be a matter of concern in ferroelectric waveguides.

Broadly speaking, a material should exhibit the following two properties in order to be insensitive to optical damage: a small density of impurities and a large dark conductivity. Evidently, a small number of impurities will prevent the excitation of a large number of free charge carriers that contribute to the photorefractive effect. A high dark conductivity inhibits the accumulation of charges in nonilluminated regions of the crystal, preventing the buildup of space charge fields that induce index changes through the electro-optic effect. (a)

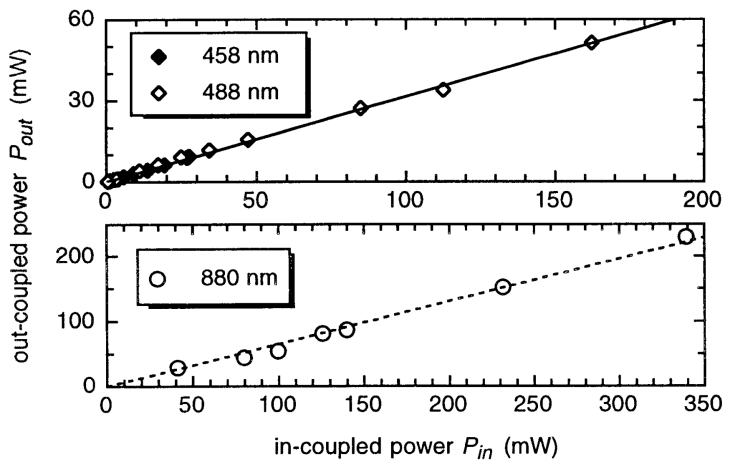

(b)
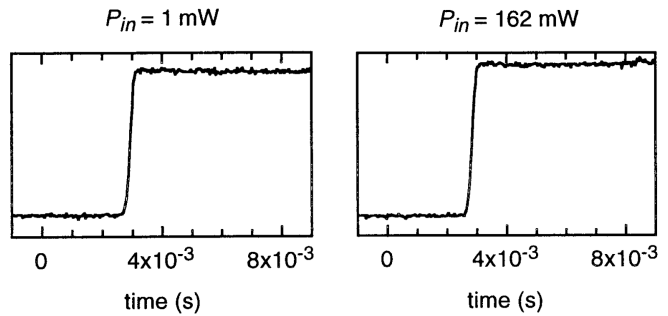

(c)
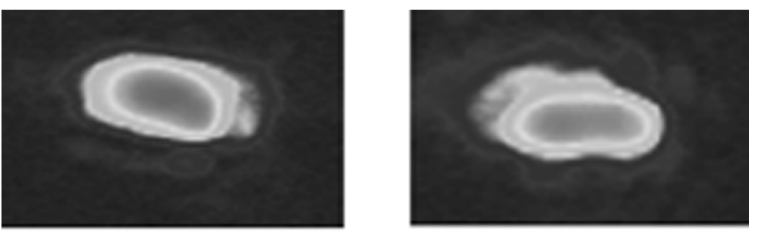

FIG. 5. Measurement of the power handling capability of a $\mathrm{KNbO}_{3}$ ridge waveguide. (a) Measured out-coupled power as a function of in-coupled power for the wavelengths of 457,488 , and $880 \mathrm{~nm}$. The curves indicate fitted linear functions. (b) Temporal evolution of the out-coupled power at $488 \mathrm{~nm}$ after switching the beam on using an electrical shutter with a switching time of $3 \mathrm{~ms}$. The measurement was done for in-coupled powers of 1 (left) and $162 \mathrm{~mW}$ (right). (c) Modal intensity profile of the out-coupled light for 1 (left) and $162 \mathrm{~mW}$ (right) in-coupled power at a wavelength of $488 \mathrm{~nm}$.

A rough method by which to detect optical damage is to observe the output near field and the output power as a function of increasing input power, usually referred to as a power handling capability measurement. At the onset of optical damage, waveguides tend to guide less power and the mode tends to show distortions. Figure 5(a) shows the power transmitted as a function of in-coupled power in the $\mathrm{TM}_{00}$ mode (polarized parallel to the $b$ axis) measured at wavelengths of 457, 488, and $880 \mathrm{~nm}$. The maximum intensities in the guide were $1.0 \times 10^{5} \mathrm{~W} \mathrm{~cm}^{-2}$ at $457 \mathrm{~nm}, 5.4 \times 10^{5} \mathrm{~W} \mathrm{~cm}^{-2}$ at 488 $\mathrm{nm}$, and $1.1 \times 10^{6} \mathrm{~W} \mathrm{~cm}^{-2}$ at $880 \mathrm{~nm}$. The maximum incoupled powers were limited by the available laser power at all three wavelengths. No sign of deviation from the expected linear behavior, indicated by fitted curves, was observed. We investigated the temporal evolution of the transmitted power after switching on the laser beam using an electrical shutter with a switching time of approximately 3 ms. Figure 5(b) shows the result of this measurement with in-coupled powers of 1 and $162 \mathrm{~mW}$, respectively, at a wavelength of $488 \mathrm{~nm}$. No sign of power fluctuation is observed after switching the beam on. Figure 5(c) displays the mode intensity profiles for these two input powers. 


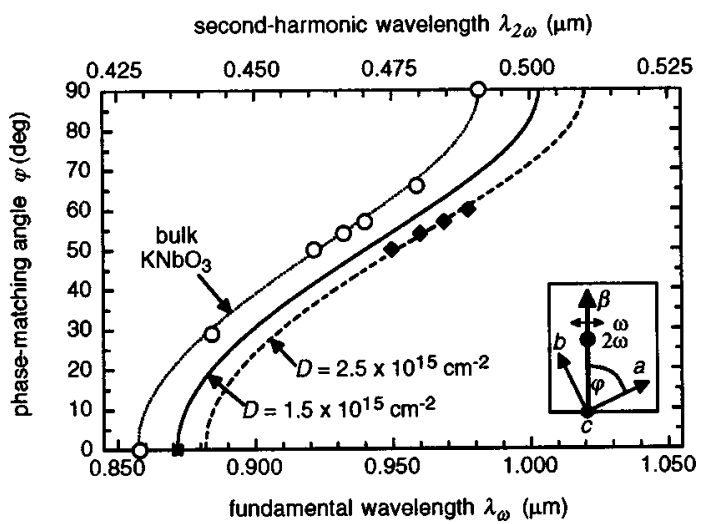

FIG. 6. Phase-matching angle as a function of wavelength at room temperature in a $\mathrm{KNbO}_{3}$ crystal $(\cdots)$ and ridge waveguide implanted with ion doses of $1.5 \times 10^{15}(-)$ and $2.5 \times 10^{15} \mathrm{~cm}^{-2}(---)$. The phase-matching angle is calculated for the $\mathrm{TE}_{00}^{\omega} \rightarrow \mathrm{TM}_{00}^{2 \omega}$. The inset displays the geometry with $\beta$ denoting the direction of propagation, $a, b$, and $c$ the crystal axes, and $\omega$ and $2 \omega$ the direction of polarization of the fundamental and secondharmonic, respectively. The data points represent measurements in differently cut crystals.

Similar power throughput measurements were carried out in annealed proton-exchanged $\mathrm{LiNbO}_{3}$ waveguides where the onset of optical damage was observed at about 5 $\mathrm{mW}$ at a wavelength of $633 \mathrm{~nm},{ }^{37}$ and in proton-exchanged $\mathrm{LiTaO}_{3}$ waveguides where throughput degradation occurred at about $50 \mathrm{~mW}$ power at $860 \mathrm{~nm} .{ }^{35}$ The authors of Ref. 37 observed an improved input-output behavior of their annealed proton-exchanged $\mathrm{LiNbO}_{3}$ waveguides after irradiation with $1 \mathrm{MeV}$ protons. The power at which optical damage started increased to $16 \mathrm{~mW}$ at $633 \mathrm{~nm}$ after ion beam irradiation and was attributed to a decreased photovoltaic current.

The results shown in Fig. 5, together with the secondharmonic stability measurement shown in Fig. 12, lead us to the conclusion that $\mathrm{KNbO}_{3}$ waveguides have excellent power handling capabilities, as no sign of optical damage was observed. We attribute this to the relatively high conductivity of $\mathrm{KNbO}_{3}$. Measurements of the conductivity of oxidic materials depend critically on various parameters such as growth conditions, impurity and defect density, and measurement conditions. Reported dark conductivity values may extend over several orders of magnitude for one material. For $\mathrm{KNbO}_{3}$, a typical value is $10^{-12} \Omega^{-1} \mathrm{~cm}^{-1}$, while for $\mathrm{LiTaO}_{3}$ and $\mathrm{LiNbO}_{3}$, well known as optical storage materials, typical values of $10^{-18}-10^{-19} \Omega^{-1} \mathrm{~cm}^{-1}$ are reported. ${ }^{38}$

\section{SECOND-HARMONIC GENERATION}

\section{A. Evaluation of phase-matching configurations}

Unlike other materials, e.g., $\mathrm{LiNbO}_{3}, \mathrm{LiTaO}_{3}$, and $\mathrm{KTiOPO}_{4}(\mathrm{KTP}), \mathrm{KNbO}_{3}$ does not require periodic poling for phase-matched blue light generation since phase matching is provided by the material birefringence. ${ }^{5}$ Figure 6 shows the phase-matching angle, defined as the angle between the direction of propagation and the $a$ axis of the crystal, as a function of wavelength at room temperature for two different ion doses. While in a bulk crystal, angle tuned second-harmonic generation in this geometry is critical with a maximum walk-off angle of $1^{0}$, the problem of walkoff is eliminated in a channel waveguide. The calculation, indicated by curves, is based on the dispersion of the effective mode indices derived in Sec. IV. For an ion dose of $D$ $=1.5 \times 10^{15} \mathrm{~cm}^{-2}$, typical of the formation of waveguides for second-harmonic generation, the curve for the $\mathrm{TE}_{00}^{\omega}$ $\rightarrow \mathrm{TM}_{00}^{2 \omega}$ interaction is shifted by about $20 \mathrm{~nm}$ with respect to the bulk crystal. Room-temperature phase matching in the waveguide is possible between 0.877 and $1.014 \mu \mathrm{m}$ whereas in a bulk crystal the range extends from 0.858 to $0.982 \mu \mathrm{m}$. Of particular interest for blue light generation is the point at $\varphi=0^{0}$ because this geometry allows the generation of the shortest blue wavelength of about $0.438 \mu \mathrm{m}$ and the use of the nonlinear optical coefficient $d_{32}$ which is the largest offdiagonal coefficient of $\mathrm{KNbO}_{3}$. Notice that in this geometry the crystal can be cut such that second-harmonic generation is provided by either a $\mathrm{TE}^{\omega} \rightarrow \mathrm{TM}^{2 \omega}$ ( $c$ axis perpendicular to the plane of propagation, $c$-cut crystal) or a $\mathrm{TM}^{\omega} \rightarrow \mathrm{TE}^{2 \omega}$ ( $b$-cut crystal) interaction. The accuracy of the calculated phase-matching wavelengths is limited by the uncertainty of the effective mode indices calculated from the refractive index profiles. The effective indices are known with an accuracy of $\pm 10^{-3}$. As a result the accuracy of the predicted phase-matching wavelengths is $\pm 2.5 \mathrm{~nm}$. The data points in Fig. 6 represent phase-matching wavelengths measured in differently cut crystals.

To obtain a high second-harmonic generation conversion efficiency in a waveguide, the phase-matching condition $N_{\omega}=N_{2 \omega}$ has to be maintained over a long distance. Besides index nonuniformities present in the original substrate crystal, additional nonuniformities can originate form the waveguide fabrication process. For example, width or thickness variations along the waveguide axis cause spatial variation of the effective mode indices as these depend on the geometrical parameters of the waveguide. Such nonuniformities reduce the effective second-harmonic interaction length and, as a consequence, the conversion efficiency. We investigated in detail the birefringence uniformity of ion-implanted $\mathrm{KNbO}_{3}$ waveguides and bulk crystals by using collinear and noncollinear second-harmonic generation. ${ }^{39}$ From these measurements we concluded that the optical uniformity of the material in the waveguiding layer is generally not affected by the ion irradiation. Regarding the geometrical parameters, a thickness variation of $1 \%$ or a width variation of $10 \%$ in a $5 \times 5 \mu \mathrm{m}^{2}$ channel guide leads to a loss of $30 \%$ of secondharmonic power compared to the case of a perfectly uniform waveguide. These tolerances can be considered as rather relaxed. The $\mathrm{He}^{+}$ion beam is defocused to a spot size of about $1 \mathrm{~cm}^{2}$ and scanned over an area of $2 \times 2 \mathrm{~cm}^{2}$ to ensure homogeneous implantation. Therefore, the thickness variation of a planar waveguide is expected to be well below $1 \%$. The precision achievable by photolithographic structuring is distinctly better than $10 \%$ so that significant birefringence nonuniformity due to width variations can be considered to be unlikely. As a conclusion, we find that the key for good birefringence uniformity is mainly a homogeneous substrate crystal. 
(a)

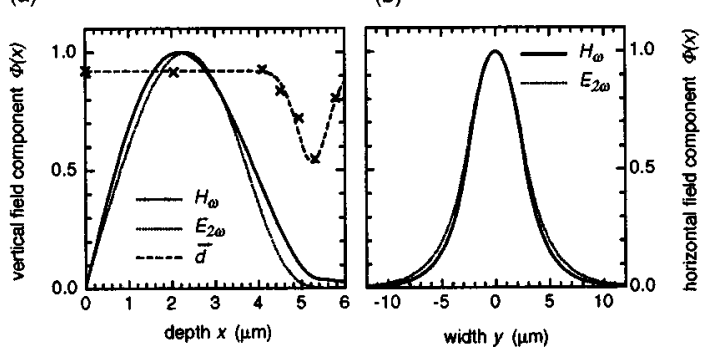

FIG. 7. Vertical (a) and horizontal (b) field components and normalized nonlinear optical coefficient as a function of depth and width, respectively, for the $\mathrm{TM}_{00}^{\omega} \rightarrow \mathrm{TE}_{00}^{2 \omega}$ interaction in a ridge waveguide. Waveguide parameters: $d=5.3 \mu \mathrm{m}, w=5.0 \mu \mathrm{m}, h=1.0 \mu \mathrm{m}$. The dashed line (---) in (a) indicates the depth profile of the normalized nonlinear optical coefficient $\bar{d}$ (the same ordinate scale as for the field).

\section{B. Calculation of overlap integrals}

The evaluation of overlap integrals is another key for the design of optimized waveguides for second-harmonic generation. To calculate the overlap integral given by Eq. (5), we use the field distributions derived by the effective index method (Sec. IV) and the depth profile of the nonlinear optical susceptibility obtained from surface second-harmonic measurements discussed in Ref. 40.

The calculation shows that only the interaction between the lowest-order modes at both wavelengths gives rise to a significant overlap integral whereas interactions with higherorder modes involved are of no practical importance. The overlap integral $\Gamma^{(00,00)}$ roughly grows inversely proportional to guide thickness $d$. For practical use only waveguides with a thickness $d>4.5 \mu \mathrm{m}$ are of interest because in thinner guides the loss is too large. ${ }^{32}$

In Fig. 7 we have plotted the vertical and horizontal distributions of the main field components of the $\mathrm{TM}_{00}^{\omega} \rightarrow \mathrm{TE}_{00}^{2 \omega}$ interaction in a ridge waveguide with $d$ $=5.3 \mu \mathrm{m}, w=5.0 \mu \mathrm{m}$, and $h=1.0 \mu \mathrm{m}$. Figure 7(a) shows the vertical components of the fundamental and secondharmonic fields together with the depth profile of the nonlinear coefficient. The modal overlap is limited to depths $x$ $<4 \mu \mathrm{m}$ where $\bar{d}(x)=0.92$ is constant. ${ }^{40}$ Figure 7 (b) displays the horizontal field components.

The channel width dependence of the overlap, plotted in Fig. 8(a) for a waveguide with $h=0.9 \mu \mathrm{m}$, is characterized by a strong increase of the overlap at small widths, maxi- (a)

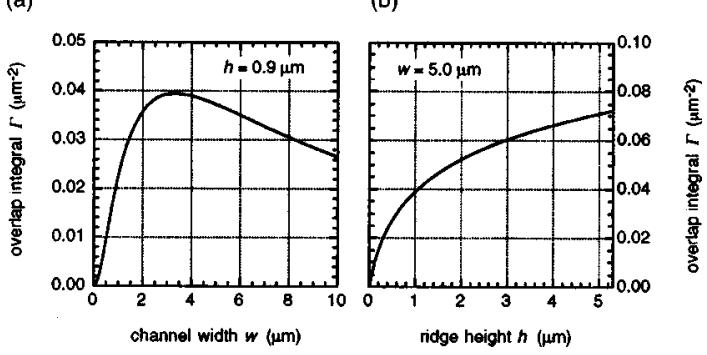

FIG. 8. (a) Overlap integral $\Gamma$ of the fundamental and second-harmonic lowest-order modes as a function of channel width. Waveguide parameters: $d=5.3 \mu \mathrm{m}, h=0.9 \mu \mathrm{m}$. (b) Overlap integral $\Gamma$ as a function of ridge height. Waveguide parameters: $d=5.3 \mu \mathrm{m}, w=5 \mu \mathrm{m}$. mum overlap for a width of $3 \mu \mathrm{m}$, and a decrease towards larger widths. The maximum of the curve is rather flat with a variation of the overlap integral of only $10 \%$ between $w$ $=2$ and $6 \mu \mathrm{m}$. This behavior can be understood by considering the horizontal components of the fundamental and second-harmonic fields as a function of the lateral coordinate $y$. For narrow waveguides $(w<2 \mu \mathrm{m})$ the field extends considerably into the surround and, as a consequence, the field distribution is broad. The minimum width of the field distribution is reached at $w=3 \mu \mathrm{m}$, and with growing channel width the field distribution broadens again. For a waveguide of $d=5.3 \mu \mathrm{m}$ thickness the maximum overlap is $0.04 \mu \mathrm{m}^{-2}$ which corresponds to an effective guide cross section of $25 \mu \mathrm{m}^{2}$. The dependence on the ridge height, displayed in Fig. 8(b) for a waveguide with $w=5.0 \mu \mathrm{m}$, shows a continuous increase due to the growing lateral field confinement with growing ridge height. However, as the ridge height approaches the guide thickness, the growth of the overlap becomes flat.

The value of the calculated overlap integral sensitively depends on the difference of the ridge and surround effective mode indices underlying the calculation of the field distributions. If, for example, one of these indices varies by 2 $\times 10^{-4}$, the overlap integral may change by as much as $30 \%$. This behavior clearly imposes a limit to the predictability of overlap integrals.

\section{Second-harmonic generation experiments}

All second-harmonic experiments discussed in Sec. VII were carried out in waveguides cut for the phase-matching configuration with $\varphi=0^{\circ}$ (Fig. 6). We recall that in this geometry the waveguide axis is parallel to $a$, the fundamental wave is polarized parallel to $b$, and the second-harmonic is parallel to $c$ (the effective nonlinear coefficient $d_{32}$ ). In the bulk crystals used in this work we measured by collinear second-harmonic generation a nonlinear optical coefficient of $d_{\text {mat }}=10.5 \mathrm{pm} \mathrm{V}^{-1}$.

The measurements were carried out with a continuouswave Ti:sapphire ring laser tunable between 0.78 and 0.92 $\mu \mathrm{m}$. The laser was oscillating with about 50 longitudinal modes. According to Ref. 41 a multimode pump leads to an enhancement of the second-harmonic power by a factor of $(2 N-1) / N \approx 2$ where $N$ is the number of independently oscillating laser modes.

We used an end-fire coupling geometry to launch the light into the waveguide with a microscope objective $(20 \times)$. Precise matching of the field distributions of the laser beam and waveguide mode is essential to obtain a high coupling efficiency. When using waveguides with an elliptical mode profile the laser beam profile was adjusted by using a prism pair with an aspect ratio of $2 \times, 3 \times$, or $4 \times$ to obtain an elliptical beam. Under optimized conditions launch efficiencies of more than $80 \%$ were achieved (the Fresnel reflection loss of $15 \%$ is not included).

The fundamental input power $P_{\omega}(0)$ was determined by measuring the fundamental power behind the coupling lens and multiplying this power by the Fresnel transmission coefficient $\left(T_{F}=0.85\right.$ for an uncoated crystal $)$ and the coupling 


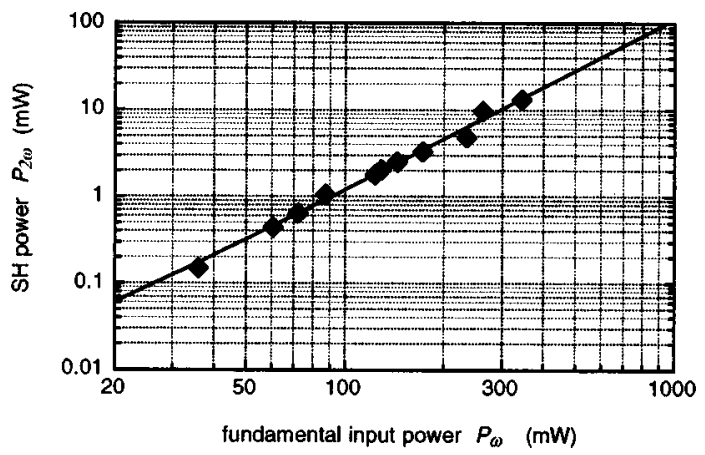

FIG. 9. Second-harmonic power as a function of the fundamental in-coupled power in a $0.73 \mathrm{~cm}$ long $\mathrm{KNbO}_{3}$ ridge waveguide. The line indicates the quadratic fit to the data. Waveguide parameters: $d=5.3 \mu \mathrm{m}, w=2.6 \mu \mathrm{m}$, $h=0.91 \mu \mathrm{m}$.

efficiency that was assumed to be $T_{C}=0.8$.

Figure 9 displays the second-harmonic power as a function of the fundamental input power measured in a ridge waveguide with the following parameters: $d=5.3 \mu \mathrm{m}, w$ $=2.6 \mu \mathrm{m}$, and $h=0.9 \mu \mathrm{m}$. A maximum blue power of 14 $\mathrm{mW}$ at $438 \mathrm{~nm}$ at room temperature was obtained with 340 $\mathrm{mW}$ of fundamental power coupled into the waveguide. In this $0.73 \mathrm{~cm}$ long guide, this corresponds to a normalized conversion efficiency of $25 \% \mathrm{~W}^{-1} \mathrm{~cm}^{-2}\left(13 \% \mathrm{~W}^{-1}\right)$. No sign of rolloff from the quadratic dependence, indicated by the line, at higher powers is observed. The fundamental power $P_{\omega}(L)$ measured at the guide end was $205 \mathrm{~mW}$. The loss at the fundamental and second-harmonic wavelengths was 0.7 and $1.3 \mathrm{~cm}^{-1}$, respectively (Fig. 3). The measured phase-matching wavelength corresponds within an accuracy of $\pm 2.5 \mathrm{~nm}$ to that predicted in Fig. 6 .

Figure 10 shows the temperature tuning curve of the second-harmonic signal measured with a fundamental power of $200 \mathrm{~mW}$. The theoretical linewidth calculated from dispersion data is $0.35{ }^{\circ} \mathrm{C}$. The measured linewidth is $0.55{ }^{\circ} \mathrm{C}$. The same linewidth broadening was measured in the substrate crystal suggesting that the distortion of the tuning curve is due to a birefringence nonuniformity already present in the crystal.

A series of measurements was carried out to evaluate the dependence of the second-harmonic conversion efficiency on

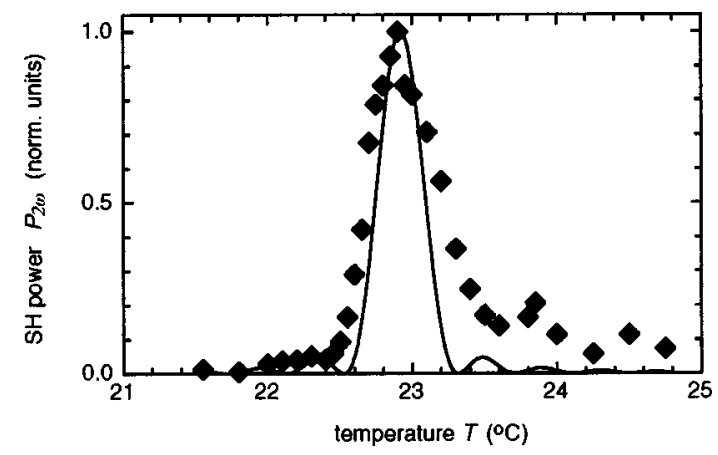

FIG. 10. Second-harmonic power as a function of temperature. The line represents the theoretical dependence calculated from refractive index data. The fundamental power was $200 \mathrm{~mW}$. (a)

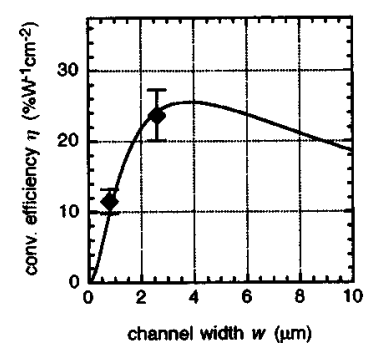

(b)

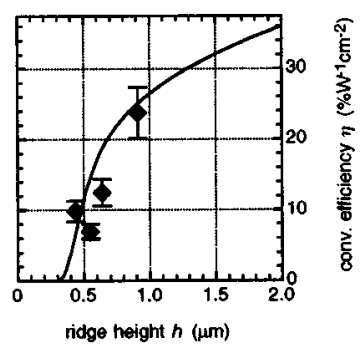

FIG. 11. (a) Second-harmonic conversion efficiency as a function of channel width. Waveguide parameters: $d=5.3 \mu \mathrm{m}, h=0.9 \mu \mathrm{m}$. (b) Secondharmonic conversion efficiency as a function of ridge height. Waveguide parameters: $d=5.3 \mu \mathrm{m}, w=5 \mu \mathrm{m}$. The lines represent the calculated functions that are based on the respective dependencies of the attenuation (Fig. 4) and overlap integral (Fig. 8).

the geometrical parameters of the guides. The calculated channel width dependence of the second-harmonic power for a ridge waveguide is shown in Fig. 11(a). The calculation is based on the channel width dependence of the waveguide loss, shown in Fig. 4(a), and the overlap integral, shown in Fig. 8(a). The strong increase of the second-harmonic power for small channel widths is due to the simultaneous decrease of the loss and increase of the overlap integral. Maximum second-harmonic power is obtained for a width of about 3 $\mu \mathrm{m}$. Towards larger channel widths, the second-harmonic power slowly decreases due to the decrease of the overlap integral which is partially compensated for by the decrease of the loss.

A series of four waveguides was produced to investigate the second-harmonic power as a function of ridge height. Figure 11(b) displays the calculated dependence, again based on the corresponding dependence of the loss, shown in Fig. 4(b), and the overlap integral, shown in Fig. 8(b). The second-harmonic power increases with increasing ridge height as the loss decreases and the overlap increases.

To investigate the stability of the blue output we monitored the second-harmonic power as a function of time for 6 $\mathrm{h}$, shown in Fig. 12. We found that the average of the second-harmonic power remained stable over the whole period and no degradation of the blue output was observed. Only the temperature of the waveguide was actively stabilized. We measured a root-mean-square signal-to-noise ratio of the second-harmonic output power of $3 \%$. This relatively

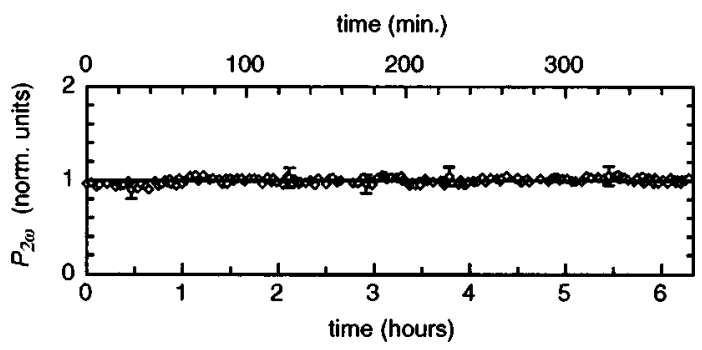

FIG. 12. Normalized second-harmonic output power as a function of time measured over a period of $6 \mathrm{~h}$. The line indicates the average secondharmonic power. The blue power was $7 \mathrm{~mW}$ with $240 \mathrm{~mW}$ of fundamental power coupled into the waveguide. 


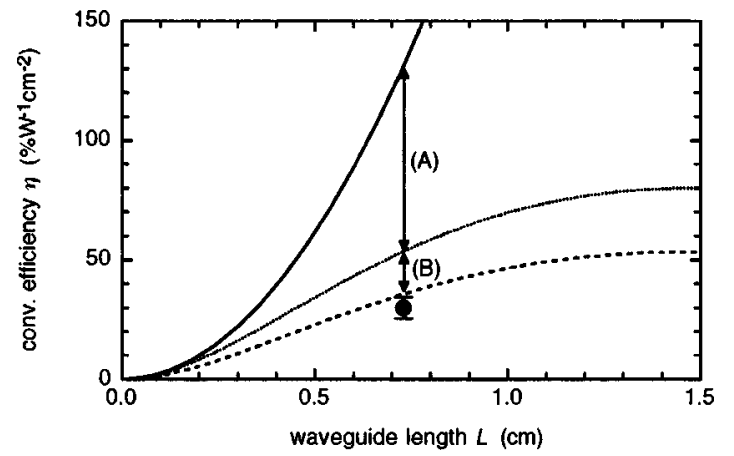

FIG. 13. Normalized second-harmonic conversion efficiency as a function of guide length calculated for the ridge waveguide that yielded the highest conversion efficiency in this work $(d=5.3 \mu \mathrm{m}, w=2.6 \mu \mathrm{m}, h=0.9 \mu \mathrm{m})$. The calculation was done on the basis of a nonlinear coefficient of $d_{w g}$ $=10.5 \mathrm{pm} \mathrm{V}^{-1}$. The dot indicates the measured value. The lines represent the following situations: $(-)$ calculation for a loss-free waveguide, $(\cdots)$ calculation assuming a loss of $\alpha_{\omega}=0.7 \mathrm{~cm}^{-1}$ and $\alpha_{2 \omega}=1.3 \mathrm{~cm}^{-1}$ as measured experimentally, (---) calculation assuming a reduced interaction length due to nonuniformity of the birefringence as observed in Fig. 9. (A) indicates the reduction of the conversion efficiency due to the linear loss, and (B) the reduction due to the index nonuniformity.

large power fluctuation is mainly due to the noise of the Ti:sapphire laser used for the measurement.

\section{DISCUSSION AND CONCLUSIONS}

The generation of $14 \mathrm{~mW}$ continuous-wave blue power with a fundamental power of $340 \mathrm{~mW}$ coupled into the waveguide is the highest continuous-wave second-harmonic conversion efficiency obtained in a $\mathrm{KNbO}_{3}$ waveguide. The conversion efficiency in the waveguide is more than five times larger than the conversion efficiency of the substrate crystal. It should be noted that the waveguide was not repoled after the implantation. As was observed in the measurements reported in Ref. 40, omitting the repoling process reduces the second-harmonic power by a factor of 2 . On the other hand, the measured conversion efficiency is enhanced by a factor of 2 because of the multimode pump laser. Therefore, the above conversion efficiency could be expected from a repoled waveguide combined with a single-longitudinalmode laser diode.

A comparison between theoretical and experimental results of a second-harmonic measurement is complicated by a number of factors, including uncertainty of the relevant material parameters such as the material nonlinearity $d_{\text {mat }}$ and the waveguide nonlinearity $d_{w g}(x, y)$, propagation loss, uncertainty of the overlap integral, and the spectral properties of the pump laser. Figure 13 shows the normalized secondharmonic conversion efficiency as a function of length calculated for a waveguide identical to the one that yielded the highest conversion efficiency reported here $(d=5.3 \mu \mathrm{m}, w$ $=2.6 \mu \mathrm{m}, h=0.9 \mu \mathrm{m})$. The dot in Fig. 13 represents the measured efficiency in this waveguide. The calculation is based on a nonlinear optical coefficient of $d_{w g}$ $=10.5 \mathrm{pm} \mathrm{V}^{-1}$ that was derived from (i) measuring the conversion in the bulk crystal of the substrate $\left(d_{\text {mat }}\right.$ $=10.5 \mathrm{pm} \mathrm{V}^{-1}$ ), (ii) considering the fact that the waveguide was not repoled ( $\bar{d}=0.7$ according to Ref. 40 ), and (iii) taking the multimode fundamental pump into account $[d($ multimode pump $) \approx \sqrt{2} d($ single-mode pump $)]$. For a waveguide of the same length without losses $(h=1)$ we calculate a conversion efficiency of $\eta_{0}=125 \% \mathrm{~W}^{-1} \mathrm{~cm}^{-2}$ (taking pump depletion into account). The losses of $\alpha_{\omega}$ $=0.7 \mathrm{~cm}^{-1}$ and $\alpha_{2 \omega}=1.3 \mathrm{~cm}^{-1}$ reduce the expected conversion efficiency to $55 \% \mathrm{~W}^{-1} \mathrm{~cm}^{-2}(h=0.38)$. The observed linewidth broadening of the temperature tuning curve (Fig. 10), present already in the substrate crystal, accounts for a further reduction of the conversion efficiency by a factor of 1.5 resulting in a expected efficiency of $37 \% \mathrm{~W}^{-1} \mathrm{~cm}^{-2}$. The remaining discrepancy between the calculation and measurement ( $\eta=25 \% \mathrm{~W}^{-1} \mathrm{~cm}^{-2}$ ) may be attributed to the uncertainty of the calculated overlap integral that is very sensitive to small variations of the effective mode indices underlying the calculation of the field distributions.

The condition $\alpha_{2 \omega} \approx 2 \alpha_{\omega}$ is well satisfied for our waveguides (Fig. 3). This allows us, according to Eq. (11), to experimentally estimate the maximum possible conversion efficiency for this waveguide. With $P_{\omega}(L)=205 \mathrm{~mW}$ we obtain $\eta_{0} \approx \tilde{\eta}=63 \% \mathrm{~W}^{-1} \mathrm{~cm}^{-2}$. Taking the aforementioned linewidth broadening due to birefringence nonuniformity and the uncertainty of the theoretical calculation into account, the measured value of $\eta_{0}$ corresponds sufficiently well to the calculated value of $125 \% \mathrm{~W}^{-1} \mathrm{~cm}^{-2}$.

It is worth briefly comparing the results of this work to our results in previous work on $\mathrm{KNbO}_{3}$ waveguides. In Cerenkov-type second-harmonic generation a blue power of $4.1 \mathrm{~mW}$ was obtained with a fundamental input power of 270 $\mathrm{mW}$ in a $1.12 \mathrm{~cm}$ long embedded channel waveguide. This corresponds to a normalized conversion efficiency of $5 \% \mathrm{~W}^{-1} \mathrm{~cm}^{-1} \cdot{ }^{17}$ (Notice that in Cerenkov-type frequency doubling the second-harmonic power grows linearly with $L$.) In the guided-mode second-harmonic experiments reported in Ref. 18, a blue power of $2.6 \mathrm{~mW}$ with an incident fundamental power of $280 \mathrm{~mW}$ was generated in a $0.58 \mathrm{~cm}$ long guide with a figure-of-merit of $10 \% \mathrm{~W}^{-1} \mathrm{~cm}^{-2}$ $\left(3.3 \% \mathrm{~W}^{-1}\right)$.

In comparison to the results reported for periodically poled $\mathrm{LiNbO}_{3}, \mathrm{LiTaO}_{3}$, and $\mathrm{KTP}$ waveguides the normalized conversion efficiency of our $\mathrm{KNbO}_{3}$ ridge waveguides is lower. This can be explained by the smaller nonlinear coefficient, the relatively high loss, and the relatively small overlap integral of these waveguides. On the other hand, the good ability to control the guide fabrication process, the excellent power handling capability, the high second-harmonic power of $14 \mathrm{~mW}$, and the stability of the second-harmonic demonstrate the suitability of $\mathrm{KNbO}_{3}$ ridge waveguides for efficient frequency conversion. For the design of further improved ridge waveguides the following four points have to be considered.

(i) The major limitation regarding conversion efficiency is due to attenuation. Possibly the loss at the fundamental wavelength could be further reduced by fabricating a wider bottom confinement barrier with two implantation steps of slightly different energy and the same total dose. A decrease of the loss at the fundamental wavelength by a factor of 2 would result in an increase of the second-harmonic power by a factor of 1.3 . 
(ii) An increase of the waveguide length from 0.7 to 1.2 $\mathrm{cm}$ would lead to an increase of the second-harmonic power by a factor of 1.4 as can be derived from Fig. 13 .

(iii) Figure 8(b) shows that an increase of the ridge height from 0.9 to $1.8 \mu \mathrm{m}$ would result in an increase of the second-harmonic power by another factor of 1.5 . Fabrication of ridges of this height is possible although at the technological limit of the presently used etch process due to the small etch rate of $\mathrm{KNbO}_{3}$.

(iv) Elimination of the index inhomogeneity would lead to an enhancement of the second-harmonic power by a factor of 1.5. This implies that the use of high quality substrates is a key to high conversion efficiency in waveguides.

Further improvement in the waveguide fabrication technology should allow generation of a second-harmonic power of $15-25 \mathrm{~mW}$ with an in-coupled power of $200 \mathrm{~mW}$ and a normalized conversion efficiency of about $50 \% \mathrm{~W}^{-1} \mathrm{~cm}^{-2}$.

\section{ACKNOWLEDGMENT}

The authors are grateful to Hermann Wüest and Jaroslav Hajfler, both with ETH Zürich, for crystal growth and expert crystal polishing. They also thank Daniel Rytz (Forschungsinstitut für Edelsteine/Edelmetalle, Germany, formerly with Sandoz Optoelectronics, France) who provided some of the crystals.

${ }^{1}$ P. Günter, Opt. Commun. 11, 285 (1974).

${ }^{2}$ M. Zgonik, R. Schlesser, I. Biaggio, E. Voit, J. Tscherry, and P. Günter, J. Appl. Phys. 74, 1287 (1993).

${ }^{3}$ Y. Uematsu, Jpn. J. Appl. Phys. 13, 1362 (1974).

${ }^{4}$ D. A. Roberts, IEEE J. Quantum Electron. 28, 2057 (1992).

${ }^{5}$ I. Biaggio, P. Kerkoc, L.-S. Wu, P. Günter, and B. Zysset, J. Opt. Soc. Am. B 9, 507 (1992).

${ }^{6}$ P. Günter, P. M. Asbeck, and S. K. Kurtz, Appl. Phys. Lett. 35, 461 (1979).

${ }^{7}$ R. Waarts, S. Sanders, R. Parke, D. Mehuys, R. Lang, S. O’Brien, K. Dzurko, D. Welch, and D. Scifres, IEEE Photonics Technol. Lett. 5, 1122 (1993).

${ }^{8}$ D. Fluck, T. Pliska, and P. Günter, Opt. Commun. 123, 624 (1996).

${ }^{9}$ W. J. Kozlovsky, W. Lenth, E. E. Latta, A. Moser, and G. L. Bona, Appl. Phys. Lett. 56, 2291 (1990).

${ }^{10}$ C. Zimmermann, V. Vuletic, A. Hemmerich, and T. W. Hänsch, Appl. Phys. Lett. 66, 2318 (1995).

${ }^{11}$ G. I. Stegeman and C. T. Seaton, J. Appl. Phys. 58, R57 (1985).

${ }^{12}$ M. Yamada, N. Nada, M. Saitoh, and K. Watanbe, Appl. Phys. Lett. 62, 435 (1993)

${ }^{13}$ M. L. Bortz, S. J. Field, M. M. Fejer, D. W. Nam, R. G. Waarts, and D. F. Welch, IEEE J. Quantum Electron. QE-30, 2953 (1994).
${ }^{14}$ K. Yamamoto, K. Mizuuchi, Y. Kitaoka, and M. Kato, Appl. Phys. Lett. 62, 2599 (1993).

${ }^{15}$ D. Eger, M. Oron, M. Katz, and A. Zussman, Appl. Phys. Lett. 77, 2205 (1995).

${ }^{16}$ Q. Chen and W. P. Risk, Electron. Lett. 32, 107 (1996).

${ }^{17}$ D. Fluck, T. Pliska, P. Günter, L. Beckers, and Ch. Buchal, IEEE J. Quantum Electron. 32, 905 (1996).

${ }^{18}$ D. Fluck, T. Pliska, P. Günter, St. Bauer, L. Beckers, and Ch. Buchal, Appl. Phys. Lett. 69, 4133 (1996).

${ }^{19}$ T. Bremer, W. Heiland, B. Hellermann, P. Hertel, E. Krätzig, and D. Kollewe, Ferroelectr. Lett. Sect. 9, 11 (1988).

${ }^{20}$ L. Zhang, P. J. Chandler, and P. D. Townsend, Ferroelectr. Lett. Sect. 11, 89 (1990).

${ }^{21}$ P. Moretti, P. Thevenard, K. Wirl, P. Hertel, H. Hesse, E. Krätzig, and G. Godefroy, Ferroelectrics 128, 13 (1992).

${ }^{22}$ F. P. Strohkendl, P. Günter, Ch. Buchal, and R. Irmscher, J. Appl. Phys. 69, 84 (1991).

${ }^{23}$ D. Fluck, P. Günter, R. Irmscher, and Ch. Buchal, Appl. Phys. Lett. 59, 3213 (1991).

${ }^{24}$ A. Yariv, IEEE J. Quantum Electron. 9, 919 (1973).

${ }^{25}$ D. Fluck, D. H. Jundt, P. Günter, M. Fleuster, and Ch. Buchal, J. Appl. Phys. 74, 6023 (1993).

${ }^{26}$ T. Pliska, D. H. Jundt, D. Fluck, P. Günter, D. Rytz, M. Fleuster, and Ch. Buchal, J. Appl. Phys. 77, 6114 (1995).

${ }^{27}$ C. Solcia, D. Fluck, T. Pliska, P. Günter, St. Bauer, M. Fleuster, L. Beckers, and Ch. Buchal, Opt. Commun. 120, 39 (1995).

${ }^{28}$ T. Pliska, C. Solcia, D. Fluck, P. Günter, L. Beckers, and Ch. Buchal, J. Appl. Phys. 81, 1099 (1997).

${ }^{29}$ I. P. Kaminow, V. Ramaswamy, R. V. Schmidt, and E. H. Turner, Appl. Phys. Lett. 24, 622 (1974).

${ }^{30} \mathrm{H}$. Kogelnik, in Integrated Optics, Topics in Applied Physics, Vol. 7, edited by T. Tamir (Springer, Berlin, 1975).

${ }^{31}$ P. D. Townsend, P. J. Chandler, and L. Zhang, Optical Effects of Ion Implantation (Cambridge University Press, Cambridge, 1994).

${ }^{32}$ T. Pliska, D. Fluck, P. Günter, L. Beckers, and Ch. Buchal, J. Opt. Soc. Am. B 15, 628 (1998).

${ }^{33}$ R. Irmscher, D. Fluck, Ch. Buchal, B. Stritzker, and P. Günter, Mater. Res. Soc. Symp. Proc. 201, 399 (1991)

${ }^{34}$ D. Eger, M. A. Arbore, M. M. Fejer, and M. L. Bortz, J. Appl. Phys. 82, 998 (1997).

${ }^{35}$ P. J. Matthews, A. R. Mickelson, and S. W. Novak, J. Appl. Phys. 72, 2562 (1992).

${ }^{36}$ M. J. Jongerius, R. J. Bolt, and N. A. Sweep, J. Appl. Phys. 75, 3316 (1994).

${ }^{37}$ E. E. Robertson, R. W. Eason, Y. Yokoo, and P. J. Chandler, Appl. Phys. Lett. 70, 2094 (1997).

${ }^{38} \mathrm{P}$. Günter and J. P. Huignard, Eds., Photorefractive Materials and their Applications I (Springer, Berlin, 1988).

${ }^{39}$ T. Pliska, F. Mayer, D. Fluck, P. Günter, and D. Rytz, J. Opt. Soc. Am. B 12, 1878 (1995).

${ }^{40}$ D. Fluck, T. Pliska, M. Küpfer, and P. Günter, Appl. Phys. Lett. 67, 748 (1995).

${ }^{41}$ J. Ducuing and N. Bloembergen, Phys. Rev. A 133, A1493 (1964). 